\title{
Excitation of Silicon Microspheres Resonances with Femtosecond Laser Fabricated Glass Waveguides
}

\author{
Hüseyin Ozan Çirkinoğlu ${ }^{\mathrm{a}}$, Ulaş Sabahattin Gökay ${ }^{\mathrm{a}}$, Ali Serpengüzel ${ }^{\mathrm{a}}$, Belén Sotillo $^{\mathrm{b}}$, \\ Vibhav Bharadwaj ${ }^{\mathrm{b}}$, Shane M. Eaton ${ }^{\mathrm{b}}$, and Roberta Ramponi ${ }^{\mathrm{c}}$ \\ ${ }^{a}$ Koç University, Microphotonics Research Laboratory, Department of Physics, Rumelifeneri Yolu, \\ Sarıyer, Istanbul 34450 Turkey; ${ }^{b}$ Politecnico di Milano, Department of Physics, Piazza Leonardo da \\ Vinci 32, Milano 20133 Italy; ' IFN-CNR, Piazza Leonardo da Vinci 32, Milano 20133 Italy
}

\begin{abstract}
Optical waveguides were fabricated with femtosecond pulsed lasers on glass and characterized by transmission measurements. Glass waveguides were later used for excitation of the whispering gallery modes in a silicon microsphere. The coupling between the silicon microsphere and the femtosecond laser inscribed optical waveguide was simulated in both $90^{\circ}$ elastic scattering and $0^{\circ}$ transmission spectra. The silicon microsphere whispering gallery modes are available for both in the transverse electric and transverse magnetic polarizations with a spectral mode spacing of $0.25 \mathrm{~nm}$. Optical resonances on silicon microsphere integrated with femtosecond laser written optical waveguides may lead to future quantum optical communication devices.
\end{abstract}

Keywords: femtosecond laser written optical waveguide, generalized Lorenz-Mie theory, microsphere, optical microcavities, silicon photonics, whispering gallery mode.

\section{INTRODUCTION}

Whispering gallery modes (WGMs) are morphology dependent resonances (MDRs), which occur on the surface of circular cavities such as microspheres. ${ }^{1}$ Optical properties of the WGMs, such as high quality factors in relatively small volumes, resulted in the integration of WGMs in various photonics applications. $^{2}$ Excitation of WGMs in microspheres depend on total internal reflection (TIR) and has been performed using prisms,${ }^{3}$ tapered fibers, ${ }^{4}$ and optical fiber half couplers (OFHCs). ${ }^{5}$ Moreover, thriving femtosecond laser micromachining techniques ${ }^{6,7}$ opens the way for more efficient, stable, and controllable excitation in more compact, and robust geometries such as femtosecond laser inscribed glass optical waveguides. ${ }^{8,9,10}$ Here, we present results for the characterization of the femtosecond laser inscribed optical waveguides in glass. We also performed the analysis of the excitation of a $500 \mu \mathrm{m}$ radius silicon $(n=3.48)$ microsphere WGMs at $90^{\circ}$ elastic scattering and $0^{\circ}$ transmission spectra excited by using femtosecond laser inscribed glass waveguides in the near-infrared optical communication bands.

Nanoengineering: Fabrication, Properties, Optics, and Devices XIII, edited by Eva M. Campo,

Elizabeth A. Dobisz, Louay A. Eldada, Proc. of SPIE Vol. 9927, 99271T · (c) 2016 SPIE

CCC code: $0277-786 X / 16 / \$ 18 \cdot$ doi: $10.1117 / 12.2239326$ 


\section{FEMTOSECOND LASER INSCRIBED OPTICAL WAVEGUIDE IN GLASS}

The femtosecond laser inscribed optical waveguide are fabricated on glass using a commercial laser with a fundamental wavelength of $1030 \mathrm{~nm}$, a pulse width of $300 \mathrm{fs}$, and a repetition rate of $500 \mathrm{kHz}$. The waveguides are written with $350 \mathrm{~mW}$ laser with a numerical aperture of 0.42 at a glass depth of $25 \mu \mathrm{m}$, and $2 \mathrm{~mm} / \mathrm{s}$ inscription speed. The mode field diameter (MFD) of the glass optical waveguide at $1550 \mathrm{~nm}$ is measured to be $17 \mu \mathrm{m} \times 22 \mu \mathrm{m}$, as shown in Figure 1 .

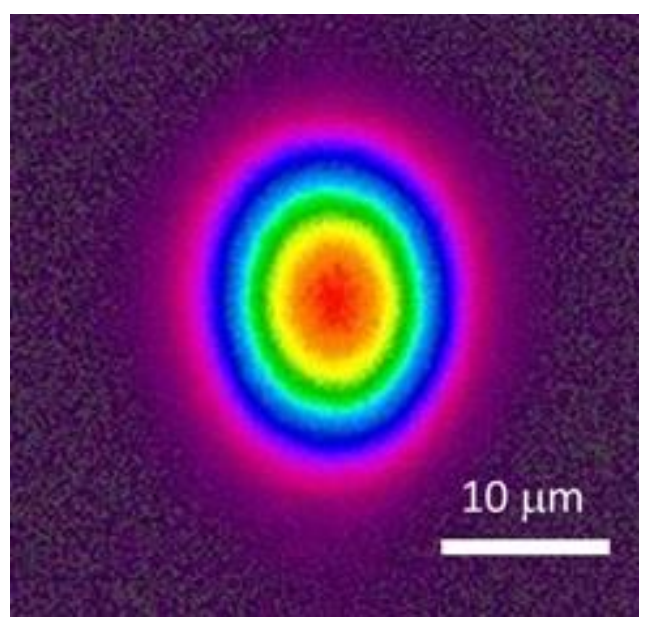

Figure 1 . Mode field diameter of $17 \mu \mathrm{m} \times 22 \mu \mathrm{m}$ for the femtosecond laser inscribed glass optical waveguide at $1550 \mathrm{~nm}$.

3. ELASTIC SCATTERING SPECTRA FROM THE SILICON MICROSPHERE

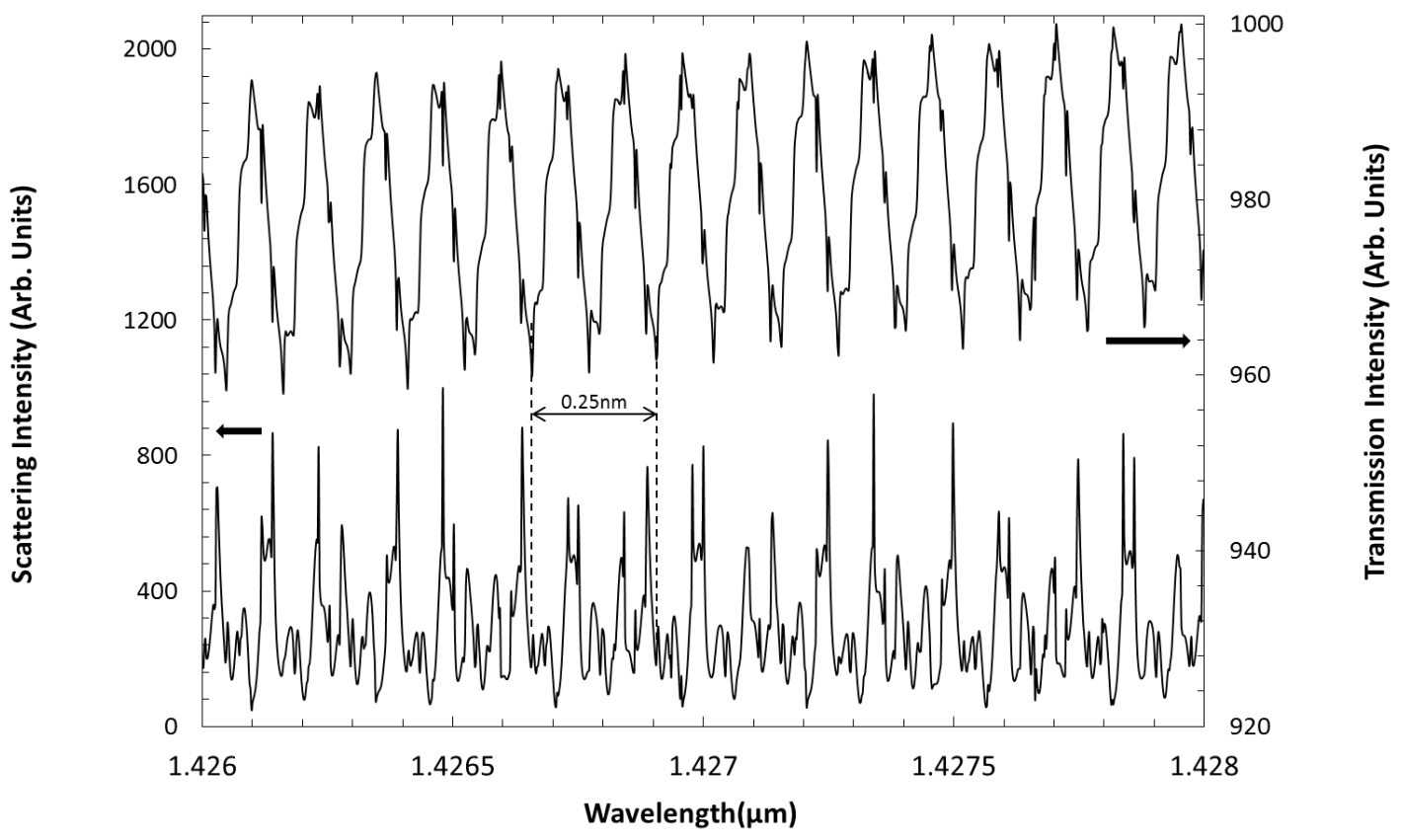

Figure $2.90^{\circ}$ elastic scattering and $0^{\circ}$ transmission transverse electric (TE) polarization spectra from the silicon sphere. 


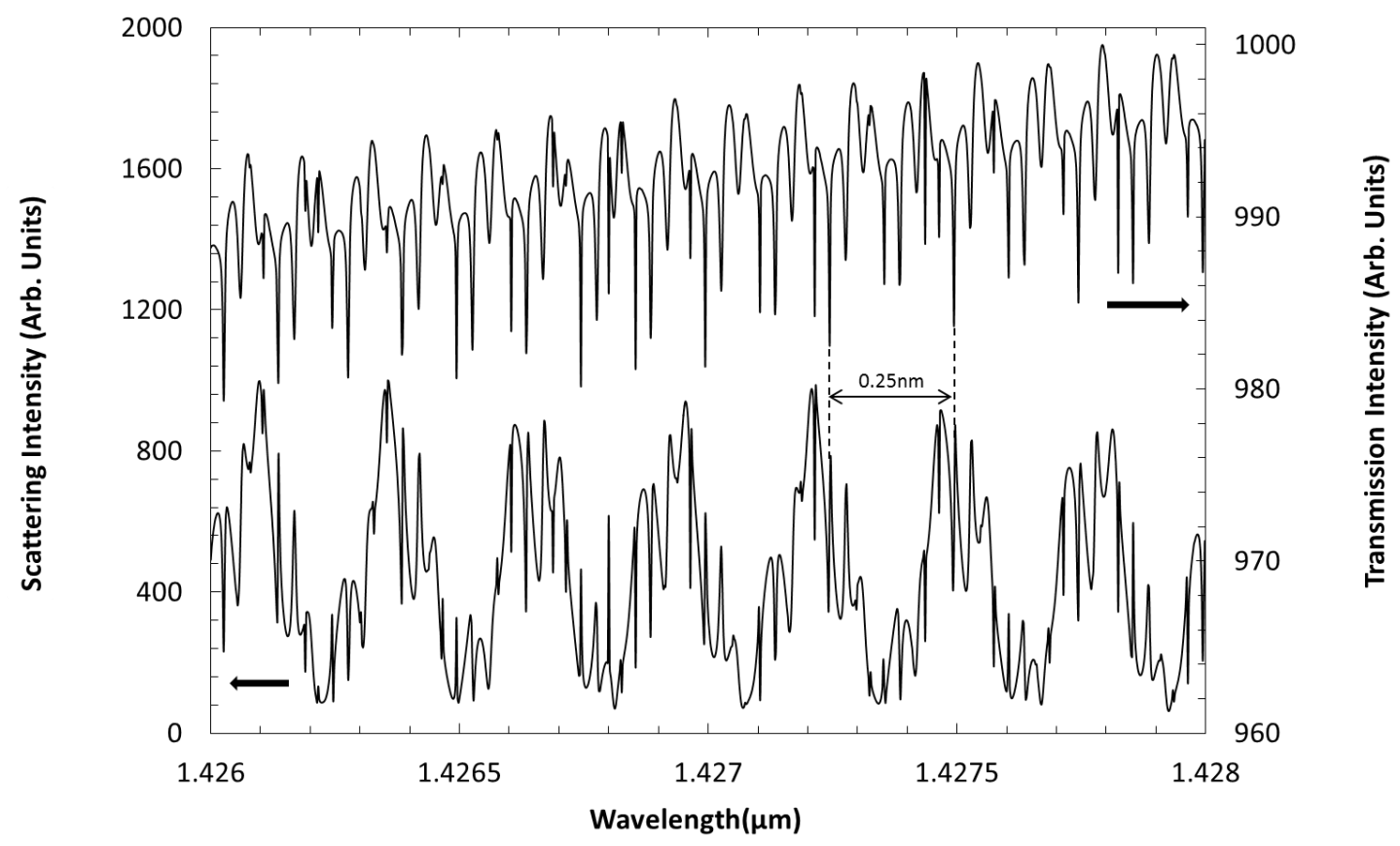

Figure $3.90^{\circ}$ elastic scattering and $0^{\circ}$ transmission transverse magnetic (TM) polarization spectra from the silicon sphere.

The $90^{\circ}$ elastic scattering and $0^{\circ}$ transmission spectra from the silicon microsphere excited with a Gaussian beam in the near-infrared telecommunication band are analyzed using the generalized Lorenz-Mie theory (GLMT) ${ }^{11}$ For a silicon sphere with a radius $a=500 \mu \mathrm{m}$ and the glass optical waveguide at a depth of $25 \mu \mathrm{m}$, the impact parameter of the excitation beam is selected as $b=525 \mu \mathrm{m}$. Due to numerical limitations, the half-width at half-maximum (HWHM) of the Gaussian beam is assumed to be $54 \mu \mathrm{m}$, which is larger than the measured MFD $(17 \mu \mathrm{m} \times 22 \mu \mathrm{m})$ of the glass optical waveguide. The near-infrared excitation laser beam is chosen to be in telecommunication band with a wavelength ranging between $1426 \mathrm{~nm}-1428 \mathrm{~nm}$. Figure 2 and Figure 3 show the numerically simulated $90^{\circ}$ elastic scattering and $0^{\circ}$ transmission spectra of the silicon microsphere for TE and TM polarizations, respectively. WGMs, observed as peaks in the $90^{\circ}$ elastic scattering spectra, correlate well with the dips of the $0^{\circ}$ transmission spectra. The $90^{\circ}$ elastic scattering and $0^{\circ}$ transmission spectra exhibit a $0.25 \mathrm{~nm}$ spectral mode spacing between two WGMs with consecutive polar mode numbers for the same radial mode order. The quality factors of the WGMs are on the order of $10^{5}$.

\section{CONCLUSIONS}

The excitation of WGMs of a silicon microsphere by using a femtosecond laser inscribed glass optical waveguide was presented. WGMs were observed in the $90^{\circ}$ elastic scattering and the $0^{\circ}$ transmission spectra for TE and TM polarizations. WGMs of the silicon microsphere with quality factors of the order of $10^{5}$ can be used in various applications such as photonic computing, sensing, 
or communication. Moreover, femtosecond laser written optical waveguides in glass are shown to facilitate the resonant excitation of circular cavities such as silicon microspheres.

\section{ACKNOWLEDGEMENTS}

We would like to acknowledge the partial support of this work by the Scientific and Technological Research Council of Turkey (TUBITAK) Grant No: $114 \mathrm{~F} 312$ and the CONCERT-Japan DiamondFab Project. We thank Center for Nano Science and Technology - Italian Institute for Technology (CNST-IIT) in Milano for access to the FemtoFab laser fabrication facility.

\section{REFERENCES}

[1] Chiasera, A., Dumeige, Y., Féron, P., Ferrari M., Jestin Y., Nunzi Conti, G., Pelli, S., Soria, S. and Righini, G. C., "Spherical whispering gallery mode microresonators," Laser Photon. Rev. 4(3), 457-482 (2010).

[2] Serpengüzel, A., Griffel, G. and Arnold, S., "Excitation of resonances of microspheres on an optical fiber," Opt. Lett. 20(7), 654-656 (1995).

[3] Gorodetsky, M. L. and Ilchenko, V. S., "High-Q optical whispering-gallery microresonators: precession approach for spherical mode analysis and emission patterns with prism couplers," Opt. Commun. 113(1), 133-143 (1994).

[4] Knight, J. C., Cheung, G., Jacques, F. and Birks, T. A., "Phase-matched excitation of whispering-gallery-mode resonances by a fiber taper," Opt. Lett. 22(15), 1129-1131 (1997).

[5] Yılmaz, Y. O., Demir, A., Kurt, A. and Serpengüzel, A., "Optical channel dropping with a silicon microsphere," IEEE Photon. Technol. Lett. 17(8), 1662-1664 (2005).

[6] Davis, K. M., Miura, K., Sugimoto, N. and Hirao, K., "Writing waveguides in glass with a femtosecond laser," Opt. Lett. 21(21), 1729-1731 (1996).

[7] Osellame, R., Taccheo, S., Marangoni, M., Ramponi, R., Laporta, P., Polli, D., De Silvestri, S. and Cerullo, G., "Femtosecond writing of active optical waveguides with astigmatically shaped beams," J. Opt. Soc. Am. B 20(7), 1559-1567 (2003).

[8] Sakakura, M., Sawano, T., Shimotsuma, Y., Miura, K. and Hirao, K., "Fabrication of three-dimensional $1 \times 4$ splitter waveguides inside a glass substrate with spatially phase modulated laser beam," Opt. Express 18(12), 12136-12143 (2010).

[9] Eaton, S. M., Zhang, H., Ng, M. L., Li, J., Chen, W., Ho, S. and Herman, P. R., "Transition from thermal diffusion to heat accumulation in high repetition rate femtosecond laser writing of buried optical waveguides," Opt. Express 16, 9443-9458 (2008).

[10] Zhang, H., Ho, S., Eaton, S. M., Li, J. and Herman, P. R., "Three-dimensional optical sensing network written in fused silica glass with femtosecond laser," Opt. Express 16, 14015-14023 (2008).

[11] Lock, J. A., "Improved Gaussian beam-scattering algorithm," Appl. Opt. 34(3), 559-570 (1995). 